\title{
Skip Metastasis to Abdominal Lymph Nodes from Lung Adenocarcinoma
}

\author{
Kensuke Nakazawa Hiroaki Satoh Morio Ohtsuka Kiyohisa Sekizawa \\ Division of Respiratory Medicine, Institute of Clinical Medicine, University of Tsukuba, Japan
}

\section{Dear Editors,}

We read with interest the article by Huang et al. (July 2007) [1] on lung cancer with isolated skip metastasis to an abdominal lymph node. We would like to share our experience regarding a patient whose condition was very similar to that reported by Huang et al. [1].

A 55-year-old woman was admitted to our hospital because of a nodular lesion that was detected incidentally by a chest radiograph. She had smoked 20 cigarettes per day for 20 years. The patient was totally asymptomatic with unremarkable physical examination. A computed tomography (CT) scan of the chest revealed a 50-mm nodule in the left upper lobe of the lung, which had invaded into the mediastinum. Pathology samples obtained by transbronchial biopsy confirmed adenocarcinoma of the lung. She received thoracic irradiation, and the response was evaluated as complete response. 4 months after completion of the irradiation, (18)F-fluorodeoxyglucose positron emission tomography (FDG-PET)/CT showed isolated lymphatic metastasis to perigastric and para-aortic lymph nodes without recurrence of lobar, hilar, or mediastinal lymph nodes. Echo-guided percutaneous biopsy confirmed lymphatic metastasis to abdominal lymph nodes. The patient received 1 course of platinum-containing chemotherapy, but the response was evaluated as progressive disease. The abdominal lymph nodes grew up to $30 \mathrm{~mm}$ in size. She died of lung cancer 2 months after the chemotherapy.

Direct lymphatic metastasis to abdominal lymph nodes without involvement of lobar, hilar, or mediastinal lymph nodes is very rare [1-3]. The condition of our patient was very similar to that of the patient reported by Huang et al. [1], and we can fully share their observations. The similar points in both patients were as follows: i) isolated skip metastasis to an abdominal lymph node from adenocarcinoma of the left lung; ii) detection of metastasis by PET/CT; and iii) pathological confirmation using specimen obtained by surgical exploration or percutaneous biopsy. PET/CT may provide useful information regarding the location of metastasis as observed in the patient reported by Huang et al. [1] as well as our patient. Although very rare, the cases reported by Huang et al. [1] and ourselves suggest that there may be a certain type of lung adenocarcinoma that metastasizes to abdominal lymph nodes without involvement of regional lymph nodes.

\section{References}

1 Huang TW, Tzao C, Chen DW, Tsai WC, Cheng YL, Lee SC: Lung cancer with isolated skip metastasis to an abdominal lymph nodes. Onkologie 2007;30:375-377.
Cerfolio RJ, Bryant AS: Distribution and likelihood of lymph node metastasis based on the lobar location of nonsmall-cell lung cancer. Ann Thorac Surg 2006;81:1969-1973.
Hubalewska-Dydejczyk A, Sowa-Staszczak A, Huszno B: Current application of sentinel lymph node lymphoscintigraphy to detect various cancer metastases. Hell J Nucl Med 2006;9:5-9.

\section{KARGER}

Fax +497614520714

Information@Karger.de

www.karger.com (c) 2008 S. Karger GmbH, Freiburg
Hiroaki Satoh, MD

Division of Respiratory Medicine

Institute of Clinical Medicine, University of Tsukuba

Tsukuba City, Ibaraki, 305-8575, Japan

Tel. +81 298533-210, Fax -320

hirosato@md.tsukuba.ac.jp 